AS TO THE NATURE OF THE PARAsites OF LEPROSY AND TUBERCUJOSIS.

\author{
BY
}

ALEXANDER G. R. FOLLERTON, F.R.C.S.,

I.ECTERER ON MACTERIOLOGY AND ON PEBLIC HFALTH TO THE MIDDLESEX HOSPITAL MEDICAL SCHOOL; COENTY MEDICAL
OFFICER OF HEALTH FOR EAST SUSSEX.

Is a recent communication Dr. Bayon, ${ }^{1}$ criticising an opinion expressed by Beauchamp Williams ${ }^{9}$ to the effect that "leprosy may be caused by, perhaps, several varieties of a highly pleomorphic streptothrix," remarks that "to suit Williams's view it "- that is to say, the parasite of leprosy - "would have to be an actinomyces also." As 't matter of fact the generic terms Streptothrix and Actinomyces, and Oospora and Nocardia also, are synonymous. The first of them is used by British pathologists zenerally, German pathologists appear to prefer usually the second, and the other two are in common use by French pathologists. A fifth generic synonym, Cladothrix, has fortunately fallen out of use. Consequently, the terms Streptothrix and Actinomyces are applicable equally as the generic thesignation of the parasite of leprosy. For there cannot be any reasonable doubt but that the parasites which Hansen identified in the tissues of lepers and described as Bacillus leprae, which Deycke was apparently the first to demonstrate in culture from leprous lesions, which Rost first succeeded in growing freely on artificial media, which Williams subsequently studied in full detail, and which have been isolated cluring the last year or two by a considerable number of bacteriologists, all belong to one and the same species (Streptothrix leprae) of "ray fungus" or streptothrix.

I have seen only stained cover-glass preparations of the acid-fast, and morphologically typical, streptothrix which Deycke obtained from cases of leprosy. But by the courtesy of Beauchamp Williams I have had opportunities of examining three strains of the organism which he has isolated from cases of leprosy occurring in Persia and India, and two strains of an identical organism which had been isolated by Rost.

It is certain that these latter parasites belong to a single species which, under certain conditions of culture on artificial media, presents a number of acid-fast rod forms which are identical with the acid-fast forms recognizable in leprous granulomata. And it is evident, I think, that this parasite is that which Deycke described under the name of Streptothrix leproides. Deycke, however, appears to have hesitated at first to accept as the essential parasite of leprosy a micro-organism which, being a streptothrix, did not conform in growth on artificial media with the then accepted morphology of Hansen's Bacillus leprac. Kedrowsky ${ }^{6}$ also recognized streptothrix forms in cultures of an organism which he isolated from a case of leprosy.

A review of modern literature concerning the parasite of leprosy shows that confusion as to the proper place in botanical classification of the specific infecting organism has been caused partly by the multiplicity of nomenclature already referred to, and partly by failure to connote the varying morphological phases of streptotricheae generally at different stages of their life-cycle. This matter was dealt with fully in the Milroy Lectures for 1910,4 and need not be referred to again, except very briefly.

The type-form of the genus Streptothrix is represented by the tangled masses of branching mycelium found in pus when suppuration has occurred in cases of streptotrichosis. Similar tufts of branching mycelium, the branching being lateral and not dichotomous, can be demonstreted nearly always in young cultures of streptotricheae in broth. The typically mycelial stage in the growth of the parasite is succeeded successively by stages of "segmentation" and "fragmentation." Rod-shaped, bacilli-like segmen's become differentiated along the length of the mycelial threads. Degeneration of intervening segments of the mycelium occurs with, or very soon after, the segmentation-so that, when fragmentation follows, the original tuft of branching mycelium is replaced by a confused aggregation of bacillary forms, some of which are long and thread-like, whilst others may appear as short bacillary or spirillar forms. The occurrence mean while of "chain sporulation" complicates the morphological picture. In cultures on artificial media aërial hyphae grow out from the surface of the mycelium, and present single chains of spores. When fragmentation occurs the aërial hyphae become separated off from the mycelium, and then present exactly the appearance of streptococci. Later these chains break up, and the spores appear as isolated spherical bodies, or in pairs. It is probable also that endogenous spore formation occurs along the length of some of the isolated rod segments.

In an old culture of a streptothrix, therefore, one finds the following elements: Longer or shorter bacillary forms, some of which may have a spiral or wavy appearance, some of which may resemble diphtheroid bacilli, and some of which may have the stump of a lateral branch still attached; short chains of spores, which resemble exactly streptococci; and paired or isolated spores. All these elements stain deeply by Gram's method; and, except when traces of branching remain-and segments showing this may be very few and far between-the surviving elements of the original mass of mycelium are indistinguishable morphologically, according to their nature, from bacilli and cocci. Under varying accidental circumstances incidental to the examination of cultures on artificial media, the spherical spores of the streptothrix may appear in stained specimens, as though they might be streptococci, diplococci, isolated cocci, or irregularly. grouped staphylococci. If a species happens to be one of the acid-fast streptotricheae, a correct morphological interpretation of specimens stained by the Ziehl-Neelsen method is obscured in another way by the fact that in many cases some only of the persistent rod-shaped or bacillary segments have acid-faist properties. Also, with most acid-fast streptotricheae, as with Koch's parasite of tuberculosis, acquisition of acid-fast properties is a matter of age. Some species which have this property develop it early and show it in nearly all of the rod segments, whilst in other species acid fast properties are acquired only after some weeks of growth on artificial media, and then the special property is manifest only in a small minority of the segments. And so it will be found that in specimens from old cultures of a typical acid.fast streptothrix-such as St. nocardii, St. eppingeri, or St. leprae-which have been stained by the Ziehl-Neelsen method, not only have "bacillary" and "coccal" forms to be considered, but the bacillary forms are, apart from casual morphological variation, of two kinds-those which are acid-fast and those which are not so.

Consideration of the varying morphology of the streptotricheae generally, and of the alternative staining characteristics of the rod forms of the acid-fast species in particular, will explain readily enough the error of those critics of Rost's earlier work who suggested that what he had described rightly as pure cultures of the parasite of leprosy were merely contaminated cultures of Hansen's acid-fast " bacillus."

Something may be added generally as to the function of the spores and persistent rod forms which in old cultures succeed the original branching mycelium of the streptothrix. With most of the numerous species which have now been examined in my laboratories, there is no possible doubt as to what happens when the organism is growing under conditions of culture on artificial media. Both the spores and the persistent rod forms of an old culture are capable, on transference to a new soil, of reproducing the branching mycelium which represents the type form of the genus. Under no circumstances of artificial culture has it appeared that rod segments are produced directly from pre-existing rod segments, and without the intervention of a mycelial stage. Equally, the spherical spores invariably sprout out into a new mycelial mass-a process which can be easily watched if an old culture of a quickly-growing species is kept for twenty-four hours in a drop of nutrient broth on a warm stage under the microscope. Also, I have found clear microscopical evidence that the rod segments of a pathogenic streptothrix may develop into a new mass of branching mycelium under the conditions of parasitic life in an animal host. In some of the more slowly-growing species the intervention of the typical mycelial stage before the development of new persistent rod forms may be difficult to demonstrate when an old culture is transplanted in a fresh medium, and especially when the culture medium used is a solid one. But, as I have explained elsewhere. this difficulty arises from the fact 
that, growth being very slow on artificial media, segmentation and fragmentation of the mycelial threads follow so closely on increasing growth that it is almost impossible in some species to trace continuously the true morphology of the organism.

In any case, it is certain that the typical streptotricheae present two distinct persistent and reproductive formsspherical spores produced under conditions of artificial culture in special spore-bearing hyphae, and rod segments. Either of these produces a new mycelium when transplanted in a fresh artificial culture medium; and the rod segments, as well as the spherical spores, are capable of producing similar mycelium in the tissues of an animal host. Further, whilst we have no evidence at present that the persistent rod forms are capable of directly reproducing themselves, as such, in growth on artificial media, there is a possibility that this direct reproducticn may occur under strictly parasitic conditions. This possibility is suggested by what is known of the biology of Sporothrix schenclii, belonging to a closely allied but somewhat higher genus of mould fungi. Some ten years ago I pointed out" that Sporothrix presented two different reproductive forms which may be termed provisionally, and for purposes of distinction, endogenous "spores" and exogenous "spore bodies." The type form of Sporothrix when growing on artificial media is represented by a tangled mass of branching mycelium which resembles generally that of Streptothrix, but which is definitely septate, and of coarser character than the other. As the culture of Sporothrix grows older provision for reproduction is made in two ways. Spherical "spores" appear along the length of, and within, the comparatively coarse mycelial thread; and ovoid, yeast-like "spore bodies" bud out from the sides of the primary mycelial threads and from the extremity of secondary lateral branches. In course of time the mycelium degenerates and, as such, disappears the only remnant of it is amorphous, Gram-staining débris. The persistent recognizable morphological elements in oldish cultures are the spherical "spores" and the yeastlike "spore bodies." If some of an old culture of Sporothrix is transferred to nutrient broth and afterwards watched on a warm stage under the microscope, it will be seen that each of the two morphologically different persistent elements gives rise to a new mycelium which sprouts out from either form, exactly as the nearly similar mycelium of a streptothrix sprouts out from either the spherical spore or the rod segment.

But whilst I have noc hitherto been able to find any evidence as to the direct multiplication of the persistent rod forms of streptotricheae in growth on artificial media, it is otherwise with the persistent forms of Sporothrix. In old cultures of Sporothrix schenckii in a nutrient broth medium there are appearances which suggest direct multi plication of the persistent yeast-like bodies. One finds in such a culture large yeast bodies, like to those which in younger cultures are seen sprouting out from the my. celium, in immediate contact with three, four, or more smaller yeast forms. Also, under the conditions of parasitic life, a direct multiplication of the yeast-like bodies, as suggested by Hektoen," appears to be indisputable. If a mouse be inoculated subcutaneously with either a young mycelial culture or with an older culture which contains only spherical and yeast forms, a slowly-extending infective infiltration of the subcutaneous tissue follows. On examination after death it is found that the area of infected granulomatous tissue is thickly crowded with Gram-staining yeast forms; but no trace of mycelium can be found. The appearances are, in short, those of a yeast infection, except that the parasites are present in much larger numbers than one finds usually in an experimental yeast infection. On transplanting these yeast forms of Sporothrix from the animal tissues in an artificial medium, a growth of the typical mycelial form occurs at once, with subsequent development of the persistent forms. It should be added that my own experiments were carried out with a culture of Sporothrix schenckii with which Hektoen had very kindly supplied me. And a statement made in the paper referred to, that " mycelium apparently does not occur under parastic conditions," applies only to experimental infection of the mouse with the particular species. Pinoy, ${ }^{8}$ on the other hand, has described recently natural cases of infection of man by Sporothrix beurmannii, in which, whilst yeast forms predominated in the tissues, mycelial forms were found in and around the capillaries of the parts infected.

In view, however, of the evidence as to the reproduction of the persistent yeast forms of Sporothrix by direct multiplication, the possibility of a similar reproduction in parasitic life of the persistent rod segments, or "bacillary" forms, of the streptotricheae has to be considered seriously. And the comparison is the more interesting when we remember that just as reliance on the morphology of one particular phase of development of the parasites of leprosy and tuberculosis as it occurs more conspicuously in the tissues has, unchecked by cultural investigation, been responsible for the inclusion of these diseases amongst the bacillary infections, so a similarly misplaced reliance on the morphological significance of a phase of the life-cycle of the parasite as it occurs in the tissues has caused error in the diagnosis of cases of sporotrichosis. It is obvious from published records that in the past certain cases of infective granulomata occurring in man, which were attributed to "yeast" or "protozoal" infection, were actually cases of sporotrichosis. The persistent yeast forms of Sporothrix in the tissues were mistaken for yeasts, or coccidia, as the case happened. It was not until Schenck succeeded in obtaining the parasite in culture, and until Hektoen added to our knowledge of it, that a correct determination of the nature of sporotrichoses was possible. Similarly Calvin, Frothingham, and Paige ${ }^{2}$ have quite recently proved, by the isolation of Sporothrix schenctiii from the infected tissues, the true nature of epizootic lymphangitis of horses, a disease which had been regarded at one time as a protozoal infection, at another time as a yeast infection.

The rod segments of streptotricheae generally representing persistent forms which are capable of reproducing the mycelial form of the parasite, and possibly also capable of direct multiplication as rod forms, the particular significance of the specially staining rod segments of the acid-fast species has to be considered next. The acid-fast properties are associated with the presence in the organism of special fatty bodies (neutral fats, fatty acids, and lipoids) whose biochemical relations have been studied especially by Much and his pupils. Much has shown that the fatty bodies are specific generally for the acid-fast group of organisms, and are capable of producing specific antibodies in the serum of infected animals. Thus the nastin obtained from the parasite of leprosy is capable of causing a powerful reaction when injected into patients with either leprosy or tuberculosis; and the similar nastin obtained from the parasite of tuberculosis will cause a reaction with patients having either infection. Much and Kössli have shown by a series of complement-fixation tests that other, and non-pathogenic, acid-fast organismsa "smegma" bacillus and the "blind worm" bacillus-are capable of reacting as antigens with the serums of tuberculous patients. Wills ${ }^{11}$ has shown, by an extended series of complement-fixation experiments, that the serums of leprous and tuberculous patients, and of lower animals which have immunized artificially against the respective parasites, contain antibodies which are specific for either organism, and also for the two non-pathogenic species, with differences which are merely quantitative. An excellent summary of the work done in the direction indicated by Much and those associated with him is given in Wills's paper, and affords strong additional evidence as to the close relationship between the parasites of leprosy and tuberculosis and certain other reputed bacilli of the acidfast kind, whose relationship with the streptotricheae has been suspected for many years.

But whilst the acquirement of acid-fast properties is associated with the presence of certain specific fatty bodies which are apparently common to all the acidfast group, it must be remembered that the possession of these properties is, in a sense, an accidental characteristic, and one which certainly depends to some extent on external circumstances. Experience with organisms such as $S t$. nocardii, St. eppingeri, St. caprae and St. leprae, shows that the acquirement of acid-fast properties during growth on artificial media is a matter of much uncertainty. Sometimes a culture will show acidfast segments within a week, or less, of the commencement of growth; in other cultures none will be found for three or four weeks. The general rule is that the older a 
particular culture is the more conspicuous are the acid-fast forms.

In the case of St. leprae it would appear that the conditions of parasitic life specially favour the acquirement of acid-fast properties; under saprophytic conditions of growth the rod segments tend to remain non-acid-fast. With Koch's parasite of tuberculosis acid-fast properties are acquired quickly during saprophytic growth; it is only in very young cultures that the non-acid-fast forms described by Klein and others can be demonstrated.

What evidence is available on the point suggests that the acid-fast forms of Streptothrix may represent not only persistent but also specially resistant elements. The spherical spores of the strepiotricheae are generally distinctly less resistant against injurious physical and chemical influences than are the spores of fission fungi; and it may be that a second resistant element-the acidfast rod form-has come into existence. Thus, Marino ${ }^{7}$ found that the acid-fast forms of the parasite of tuberculosis preserved their vitality for a considerable time in the intestinal canal of the leech, resisting the action of the digestive juices for as long as fifteen months. At the end of that time there was marked attenuation of virulence; but still inoculation of the parasites into the guinea-pig was followed in some cases by a very slow infection which resulted in death. There is also evidence of a probably similar kind with regard to the parasite of leprosy. It has been suggested that growth of this organism on artificial media is favoured in symbiosis with intestinal amoebae, and Beauchamp Williams ${ }^{10}$ has recorded his own experiments bearing on this question. With certain strains of his streptothrix the acid-fast forms appeared to die out in the course of successive subcultures. Then subcultures which appeared to consist of non-acid-fast diphtheroid rod segments were mixed with amoebae. At the end of forty-eight hours the amoebae were found to be full of acid-fast rod seyments. But an explanation other than that which supposes favourable symbiotic influences may be suggested. There is evidence that bacteria form a part of the natural food of the intestinal amoebae, and of Amoeba dysenteriae in particular. And under the circumstances it would seem most likely that the amoebae digest and assimilate the non-acid-fast segments of the streptothrix, and that in the struggle for prolonged existence other, more resistant, acid-fast forms survive, as Marino's experiments have shown to be the case with the acid-fast form of the parasite of tuberculosis which is resistant against the digestive ferments of the leech.

REFERENCES.
$1 \mathrm{H}$. Bayon, Demonstration of Specimens Relating to the Culture of the Leprosy Bacillus, BRITIsH MEDICAL JouRnaL, November 11 th. 1911, p. 1269. '2 Calvin, Frothingham. and Paige: Sporothrix and Epizootic Lymphangitis, Journ. of Med. Research., 1910, vol. xxiid,
p. 137. 3 A. G. R. Foulerton. On the Morphology and Pathogenic Action of Sporothrix schenckii, Trans. of the Path. Soc. of London, 1901, vol. lii, p. 259. ${ }^{4}$ A. G. R. Foulerton, The Streptotrichoses and cutaneous Abscesses caused by Sporothrix schenckii, a New Pathogenic Fungus, Journ. of Exper. Med., 1900, vol. v, p. 77. $6 \mathrm{~W}$. J. Kedrowsky, Zeitschr. f. Hygiene und Infektionslirank., 1910, vol. 1xvi, p. 1. 7 F. Marino, Compt. rend. de la Soc. de Biologie, 1911, vol. lxxi, p. $220 .{ }^{8}$ E. Pinoy, Forme de Sporotrichum beurmannii dans les lésions humaines. Sa fructification à l'intérior des capillaires, Compt. rend. de l'Acad. des Sciences, 1911, vol. clii, p. 286. 9 Beauchamp Williams, Leprosy, Supplement to the Indian Med. Gaz., May 1911. 10 Beauchamp December A Lecture on Leprosy, BRITIsH MEDICAL JodRNAL, Acid-fast Bacilli, Centralbl. $f$. Bakt., Abt. I, Orig., 1911, vol. 1xi,
p. 37 .

AT a recent meeting of the Society of Tropical Medicine and Hygiene Dr. Stannus read a paper on pellagra in Nyasaland, in which he brought forward strong evidence of the existence of the disease in that region; and Dr. Sandwith described the work donc in different States of America on the same disease. In a discussion that followed Drs. Sambon and Chalmers related the observations, already described in the JournaL (December 16th, 1911 , p. 1613), made during the tour in pellagra-infected parts of Italy, Roumania, Hungary, the Tyrol, France, and Spain. Dr. Sambon believes that flies (Simulium) play a part in the spread of the disease, and the epidemiological features encountered during the tour just mentioned lead Dr. Chalmers to take the same view. The trend of opinion seems to be to some such idea, but an enormous amount of work must still be done to prove this. Already, however, it seems proved that maize is not the operative cause of the disease, and this of itself constitutes a very important step forwards in the etiology of pellagra.

\section{A CASE OF ACTINOMYCOSIS OF THE LUNG.}

\section{By FRANK NICHOLSON, M.D.LOND.,}

gENIOR PHYSICIAN, HULL ROYAL INFIRMARY.

A young farm labourer from Holderness, aged 19, was: admitted into the Hull Infirmary under my care on September 22nd, 1911, and died on September 30th. He gave an indefinite history, but for a year he had apparently had a cough and expectoration with night sweats and dyspnoea, the sputum being generally thick, yellow, and offensive. In April last he had had pleurisy, he said.

The family history was unimportant. Both parents are living and well, and he had four sisters and three brothers who are all well but not very robust. His own previous history was one of good health, and he had lived in the country doing farm work since he left school.

Before admission he had had little medical treatment, but for the preceding fortnight he had been under the care of $\mathrm{Dr}$. Simpson of Skirlaugh, who kindly wrote to me. During this fortnight Dr. Simpson tells me the temperature was at first $102^{\circ}$, with poin in the $102^{\circ}$, wh pain in the knees and ankles, but subsequently did not night and morning and a considerable quantity of offensive night and mor

On admission into hospital on September 22nd the cough was very troublesome and the sputum very profuse, fetid, an purulent. Temperature, $102^{\circ}$; pulse, 102 ; respirations, 28. At th right base posteriorly there was dullness up to the angle of the scapula, with diminished fremitus, feeble breath sounds, a few. râles, and vocal resonance altered but little. The physical signs at the apex and in front, as also those over the left lung, back and front, were natural. The urine had a specific gravity of 1030, and contained no albumen, sugar, or other abnormal ingredients. The examination of the abdomen was negative. An exploring needle was inserted over the right base behind in three places, but with negative results.

During the next few days therc was nothing to report, except

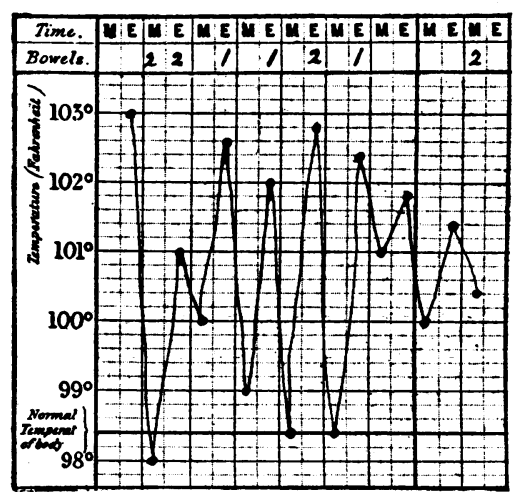

that he seen weakerand romited once, on September 25 th No tubercle bacilli were found in the sputum.

Phthisis seemed excluded, as there were no tubercle bacilli in the sputum, which was very free and purulent, and an ordinary empyema was negatived, as vibrations, though diminished, were still present at the right base, and an exploring syringe drew nothing. M y opinion was that he had an abscess of the lung or a localized enipyema between the lobes.

As he was getting worse and a fatal termination seemed certain, a needle was again inserted in two places over the right base, but drew no pus. I asked my surgical colleague to excise one or two ribs, and attempt to evacuate the abscess. One inch of the ninth rib was accordingly excised. The lung was found solid, and there was a little pus in the right pleura. The patient unfortunately collapsed, and died on the table.

At the post-mortem examination the lower lobe of the right lung was consolidated, and on section presented the appearance of grey hepatization with multiple small abscess cavities of grey hepatization with multiple small abscess cavitie scattered throughout-they were none of them larger than a large pea. As the appearance suggested actinomycosis, I had sections prepared which showed numerous small abscess cavities surrounded by dense leucocytic infiltration and were masses of Gram-staining streptothrix having the character were masses of Gram
of the ray fungus.

Actinomycosis affected the lung in about 15 per cent. of the recorded cases, but it is quite possible it is a much more common disease than is generally believed, a certain number being looked upon as non-tuberculous phthisis.

In the Journal of the American Medical Association for November 4th, 1911, Dr. Bridge of Los Angeles gives details of 17 cases. In some of these there was a mixed infection, tubercle bacilli being found in the sputum, and in most of them the sputum was noted as being very fetid.

Unless the little grains of actinomyces be found in the expectoration-they have a dirty grey and yellowish appearance, looking very like the roe of the herring-or

* Read at the meeting of the East York Division. 